\title{
$\mathrm{AIP} \mid \begin{aligned} & \text { Physics of } \\ & \text { Fluids }\end{aligned}$
}

\section{Pre-switching bifurcation of a slender jet}

P. Audier, D. Sciamarella, and G. Artana

Citation: Physics of Fluids 28, 017103 (2016); doi: 10.1063/1.4939711

View online: http://dx.doi.org/10.1063/1.4939711

View Table of Contents: http://scitation.aip.org/content/aip/journal/pof2/28/1?ver=pdfcov

Published by the AIP Publishing

\section{Articles you may be interested in}

Flow control of an elongated jet in cross-flow: Film cooling effectiveness enhancement using surface dielectric barrier discharge plasma actuator

Appl. Phys. Lett. 108, 084103 (2016); 10.1063/1.4942606

Mechanisms for laminar separated-flow control using dielectric-barrier-discharge plasma actuator at low Reynolds number

Phys. Fluids 27, 117101 (2015); 10.1063/1.4935357

Distributed forcing flow control in the wake of a blunt trailing edge profiled body using plasma actuators

Phys. Fluids 27, 035110 (2015); 10.1063/1.4914406

Control of a jet-in-cross-flow by periodically oscillating tabsa)

Phys. Fluids 24, 055107 (2012); 10.1063/1.4719150

Hysteretic mode exchange in the wake of two circular cylinders in tandem

Phys. Fluids 18, 084104 (2006); 10.1063/1.2227045

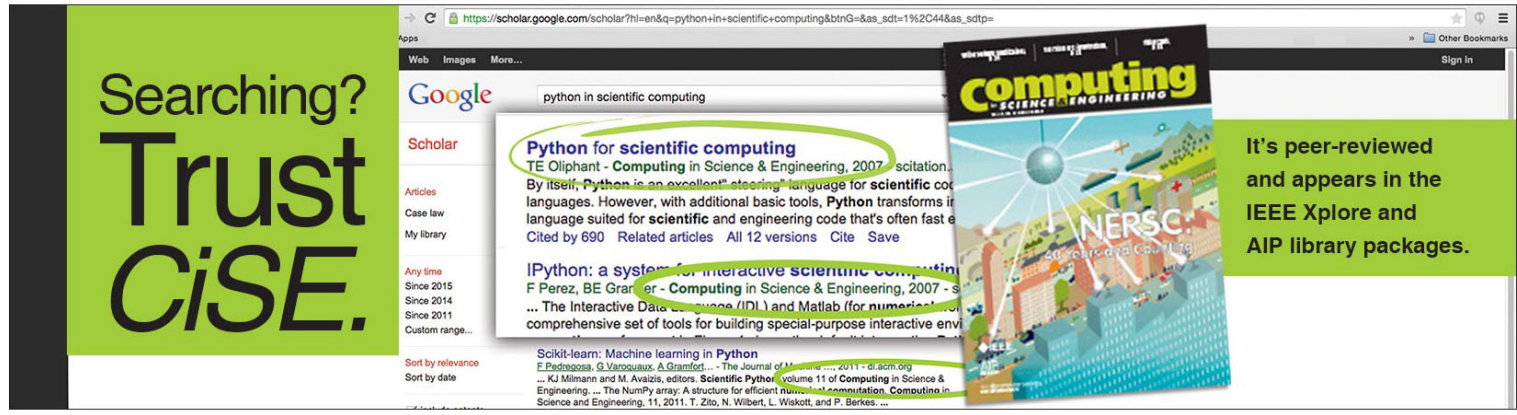




\title{
Pre-switching bifurcation of a slender jet
}

\author{
P. Audier, ${ }^{1}$ D. Sciamarella, ${ }^{2}$ and G. Artana ${ }^{1, a)}$ \\ ${ }^{1}$ LFD, Facultad de Ingeniería, Universidad de Buenos Aires-CONICET, \\ Av. Paseo Colón 850, C1063ACV Buenos Aires, Argentina \\ ${ }^{2}$ LIMSI-CNRS, Bâtiment 508, Université Paris-Sud, 91403 Orsay, France
}

(Received 3 July 2015; accepted 14 December 2015; published online 21 January 2016)

\begin{abstract}
In this work, we study the near-field of the jet flow exiting a slot-model with aspect ratio 7.5:1. The core of the slender jet separates into two streams which subsequently merge recomposing a single core jet. Axis switching occurs downstream following self-similarity rules. In order to unveil the $3 \mathrm{D}$ dynamics of this pre-switching bifurcation, stereo-PIV (Particle Image Velocimetry) measurements are performed and a phase-locking technique is implemented using surface dielectric barrier discharge plasma actuators. The device forces the flow with low-amplitude localized disturbances to produce a lock-on phenomenon. The symmetric modes of the Crow instability, developing between the counter-rotating vortex tubes formed at the slot exit, are found to account for the bifurcation process. (C)2016 AIP Publishing LLC. [http://dx.doi.org/10.1063/1.4939711]
\end{abstract}

\section{INTRODUCTION}

Jets exiting from slender slots appear in several problems of biological and technological relevance. In nature, this kind of jets can be found, for instance, in the exhalation of mussels ${ }^{1}$ or in the glottis during phonation. ${ }^{2}$ In industrial applications, rectangular jets are often used as flow control devices to improve combustion efficiency, noise suppression or mixing, ${ }^{3-5}$ as well as heat transfer enhancement. ${ }^{6}$

The initial aspect ratio of the jet is determined by the disparity between the length and width of the slot. Downstream, the jet evolves changing its cross section and interesting phenomena such as axis switching (the minor axis of the jet cross section becoming the major axis) or bifurcation (the splitting of the jet core into two streams) may take place. Axis switching is a well-known feature of non-circular jets. Bifurcation has been observed with strongly excited round jets, ${ }^{7}$ but the case of slender nozzles, where bifurcation can occur without forcing, has received much less attention. Bifurcation of round and slender jets are distinct phenomena, not only because in round jets the two streams may become completely separated but also because the vortex structures are very different.

Diverse scenarios may appear depending on the nozzle geometry and on the flow rate. Previous studies recapitulated in the review by Gutmark and Grinstein ${ }^{6}$ indicate cases presenting only axis switching, only jet splitting or a sequence involving both phenomena, with axis-switching followed by a downstream bifurcation (post-switching bifurcation). The dynamics of the post-switching bifurcation was experimentally studied for a $4: 1$ aspect ratio rectangular jet, by exciting the jet acoustically at the frequency of the preferred mode. ${ }^{8}$ The sequence of events found by the authors could be corroborated in numerical simulations of a jet with the same aspect-ratio, suddenly started and interrupted to obtain the roll-up of a single vortex structure. ${ }^{9}$

In the scenario in which bifurcation precedes axis-switching (pre-switching bifurcation), the jet core is observed to split, recompose, and switch axes further downstream. This behavior is retrieved, without any kind of excitation, in the time averaged fields of rectangular jets with aspect ratio larger than a value of about 5 . In the literature, this has been sometimes reported as jets with saddle-back axial velocity profiles. ${ }^{10}$ Even if this phenomenon was observed more than 40 years ago, ${ }^{11}$ the mechanisms involved in this kind of bifurcation process are not yet fully understood.

a)gartana@fi.uba.ar 
The saddle back velocity profile has been associated to different factors like the effect of transverse vorticity or corner vortices. ${ }^{10}$ Particularly, an analysis based on the mean transport vorticity equation has recently been performed. Other works appear in the literature that discuss the source of saddle back profiles in relation to the mixing process in the two directions perpendicular to the streamwise axis. ${ }^{12}$

Explaining this phenomenon on the basis of time averaged magnitudes is not a simple task. In particular, a detailed experimental study characterizing the full field dynamics in the near-field of the jet and identifying the evolution of individual vortex structures seems to be missing. ${ }^{10}$ Our investigation is a contribution to unveil the dynamics of these individual structures and throw light on this matter. The experiments in this work lead to the identification of a mechanism that has not been invoked before: the development of the instability of two initially parallel vortex tubes formed at the jet exit.

Our study focuses on the volumetric flow phenomena encountered in a continuous and unconfined air jet. The nozzle has the form of two facing cylinders defining a rectangular cross section at the neck, with an aspect-ratio of 7.5:1. The design of this constriction, used in the past in numerous phonation studies, ${ }^{13-16}$ is a particularly attractive geometry to study slender jets, mainly because of its simplicity. In order to have experimental access to the 3D dynamics of the pre-switching bifurcation phenomenon, we resort to the slight and localized excitation produced by surface dielectric barrier discharge (DBD) devices. The actuation is used to produce a lock-on phenomenon that allows for phase-averaging of the stereoscopic PIV measurements we perform.

The article is organized as follows. The experimental setup is described in Section II and results in Section III. Time averaged flow field measurements of unexcited jets are documented in Section III A. Section III B contains the results of the phase-locked measurements followed by a detailed discussion of the involved phenomena (Sec. III C). Conclusions are provided in Section IV.

\section{EXPERIMENTAL SETUP AND METHODS}

\section{A. Nozzle model}

The jet discharges into a quiescent atmosphere from a constriction formed by two half-cylinders disposed as shown in Fig. 1. These two "lips" define an aperture with a rectangular cross section of $15 \mathrm{~mm} \times 2 \mathrm{~mm}$ at the neck. The associated aspect ratio is of 7.5:1 and the hydraulic diameter $D_{h}=4 A_{0} / p \simeq 3.5 \mathrm{~mm}$, where $A_{0}$ is the exit cross section and $p$ is the perimeter. The model is built with a 3D printer using a polylactic acid polymer (PLA). This material has dielectric properties suitable to support the use of plasma actuators. The constriction is composed of the two antisymmetrical parts shown in Fig. 2(b). This choice is made to set the aspect-ratio of the slot precisely and to simplify the installation of plasma actuators.
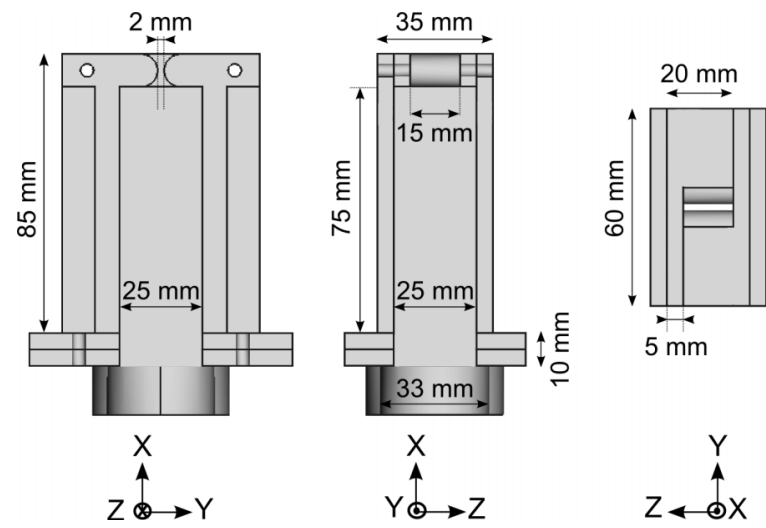

FIG. 1. Schematic diagram of the model. Cross section planes and top view. 


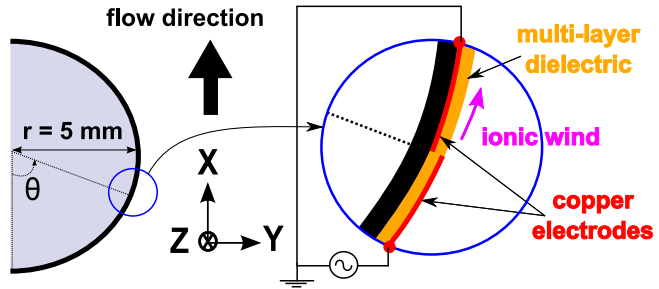

(a)

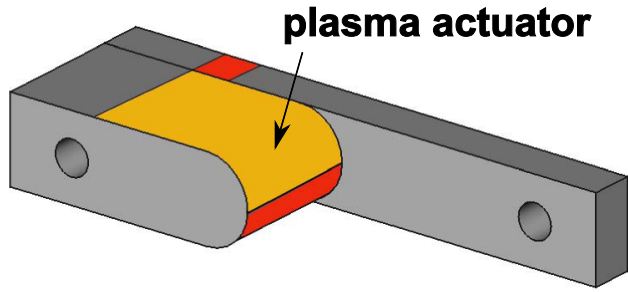

(b)

FIG. 2. Schematic diagram of one actuator installed on one half-part of the nozzle. Cross section view (a) and perspective view (b).

\section{B. Plasma actuators}

For the last 15 years, surface DBD devices have settled as one of the most sophisticated actuator devices for flow control. Unlike other type of devices (e.g., acoustic), plasma actuators enable to produce a localized perturbation, close to the surface of the body, and with a great versatility (a wide range of amplitudes and frequencies of perturbations operated through the driven electrical signal). DBD plasma actuators have demonstrated their authority to manipulate the dynamics of different flows in a wide range of applications. ${ }^{17-19}$ In the case of our geometry, plasma actuators can be used to excite the preferred mode of the jet. The preferred mode of an unexcited jet is the one with the largest growth rate: this means that it is the most recurrent, prevailing on average over the others. By exciting the jet with a slight perturbation having a frequency close to the one of the preferred mode, it is possible to drive the jet with a cyclic behavior (lock-on) and define a clear phase. This kind of excitation removes randomness and organizes the coherent structures. The dynamics of the "clean" driven jet is thus expected to be representative of the most recurrent dynamics in the natural unexcited flow. Thanks to phase-locking, the dynamics of the 3D vortical structures can be derived by suitably combining the measurements of different slices at the different phases of the cycles.

Typically, a surface plasma actuator operates by applying a high voltage between two electrodes separated by a dielectric material. This set is disposed on the surface of the geometry with one of the electrodes exposed to the atmosphere. Due to the strong electric field, a non-thermal plasma is created above the dielectric surface. By the mechanism of ion collision, a momentum exchange occurs between charged particles and neutrals inducing an electrohydrodynamic (EHD) force. The forcing takes place mainly in a small region close to the edge of the exposed electrode where the electric field is more intense. The result is a wall tangential jet flow, called ionic wind. As the amplitude and the frequency of the excitation are directly linked to the imposed electrical signal, well-defined flow perturbations can be introduced to perform a specific control strategy.

The actuators are disposed as illustrated in Fig. 2. They consist of two copper electrodes $(0.10 \mathrm{~mm}$ thick) mounted asymmetrically on both sides of a dielectric sheet composed of two layers of polyamide film, representing a total thickness of $0.32 \mathrm{~mm}$. The electrodes are $15 \mathrm{~mm}$ large in order to obtain an actuation all along the slot length. The plasma is produced by applying a sinusoidal signal to the air-exposed electrode with a high-voltage amplitude $\mathrm{V}_{H V}=2.5 \mathrm{kV}(5.0 \mathrm{kV}$ peak to peak $)$ and a frequency $\mathrm{f}_{H V}=8 \mathrm{kHz}$. A signal generator is used to create the sinusoidal signal. The high-voltage is provided by amplifying this signal with an audio amplifier combined with a car ignition coil. The position of the active air-exposed electrode can be established by the angle $\theta$, as illustrated in Fig. 2(a) for a single actuator. In our experiment, we set $\theta \simeq 80^{\circ}$ in order to guarantee that the actuation is performed upstream flow separation. Each plasma actuator is installed on one half-cylinder part as shown in Fig. 2(b). The position and the geometry of both actuators are identical and symmetrical compared to the $x z$ symmetry plane. Both are operated simultaneously with the same driving signal.

To impose a periodic actuation at the desired frequency, a burst modulation is applied to the sinusoidal signal switching the actuators on and off with a frequency $\mathrm{f}_{\text {burst }}{ }^{20}$ Such modulation is implemented in our setup by multiplying the carrier sinusoidal signal $\left(\mathrm{f}_{H V}\right)$ by a square signal $\left(\mathrm{f}_{B M}\right)$. The duty-cycle is fixed at $50 \%$. Note that the excitation is not sinusoidal and therefore harmonics of $\mathrm{f}_{B M}$ are also present in the fluid dynamic modulation. The ionic wind velocity forces the flow as a thin 


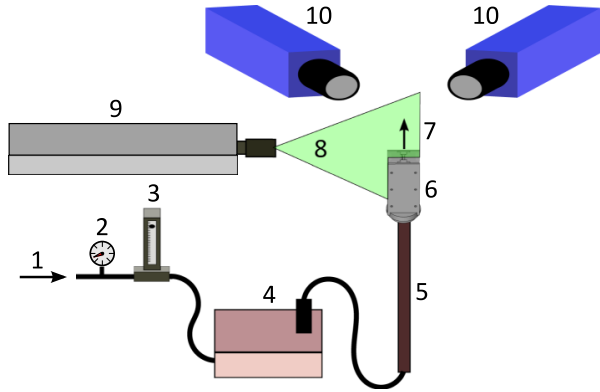

(a)

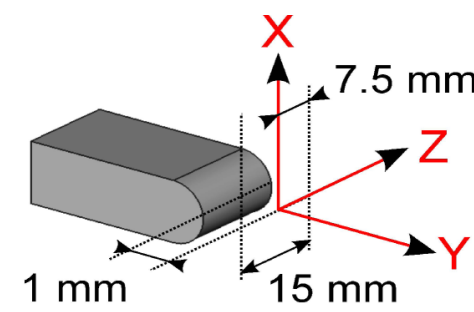

(b)

FIG. 3. Schematic diagram of the general experimental arrangement with (1) pressurized air input, (2) manometer, (3) flowmeter, (4) seeding system, (5) circular pipe, (6) model, (7) seeded flow, (8) light sheet, (9) double-pulsed laser, and (10) two cameras in side scattering arrangement (a). Location of the origin for SPIV measurements (b).

wall jet ${ }^{21}$ with a velocity around $2 \mathrm{~m} \mathrm{~s}^{-1}$. The ratio of momentum incorporated by the plasma and the momentum of the unexcited jet was estimated to be lower than $10^{-2}$.

\section{Arrangement of the experimental setup}

The arrangement of the experimental setup is shown in Fig. 3. Compressed air is supplied by the laboratory network and adjusted with a pressure regulator. The airflow is monitored with a flow-meter and seeded with micron sized synthetic oil Di-Ethyl-Hexyl-Sebacat (DEHS) droplets, sprayed by a smoke generator seeding system. The pneumatic system fed a circular pipe leading to the model, which is mounted on a moving platform. An extractor hood, operated at a low velocity and placed $1 \mathrm{~m}$ away from the test area, prevented the room from smoke filling and avoided their inhalation. A double-pulsed Nd:YAG laser $(532 \mathrm{~nm} / 135 \mathrm{~mJ})$ is combined with laser optics to form a light sheet parallel to the streamwise direction of the flow. Images are acquired with two ImagerProX2M cameras fitted with $60 \mathrm{~mm}$ lenses combined with Scheimpflug adaptors. The image size is $58 \mathrm{~mm} \times 34 \mathrm{~mm}$ with a spatial resolution of $34.7 \mu \mathrm{m}$ per pixel. In this experiment, each camera is set with an angle of $45^{\circ}$ with the laser sheet plane, resulting in an angle of $90^{\circ}$ between the two camera planes. This arrangement is chosen to minimize the out-of-plane error. ${ }^{22}$

The whole system enables the capture of flow slices at different locations and/or with different orientations, without involving new optical alignments, focusing or calibrations. It is possible to displace the platform along the $z$-axis through micro-metric screw drives with an accuracy of $1 \mu \mathrm{m}$. For each study case, a number of 36 series of $x y$ slices are acquired to perform a subsequent volumetric reconstruction of the flow. The separation between slices is not constant along the slot length: it is densified close to the jet axis and more separated as $z$ is increased; it ranges from $0.5 \mathrm{~mm}$ to $1 \mathrm{~mm}$. The slot is positioned at the channel exit to generate a free jet without any confinement. The first available measurement points are located at about $5 \mathrm{~mm}$ away from the $\mathrm{x}$-axis origin $\left(1.5 x / D_{h}\right)$, located as indicated in Fig. 3(b). The flow rate is adjusted to a constant value in the range from $18 \mathrm{lpm}$ $\left(300 \mathrm{~cm}^{3} \mathrm{~s}^{-1}\right)$ to $26 \mathrm{lpm}\left(433 \mathrm{~cm}^{3} \mathrm{~s}^{-1}\right)$, corresponding to mean exit velocities (the mean bulk velocity at the neck) from $10 \mathrm{~m} \mathrm{~s}^{-1}$ to $14.5 \mathrm{~m} \mathrm{~s}^{-1}$. For these flow rates, the nozzle dimensions yield Reynolds numbers (based on the hydraulic diameter $D_{h}$ and the mean velocity at the neck) ranging from 2300 to 3200 .

\section{Acquisition and post-processing}

In each experiment, acquisition of the 3D velocity vectors is carried out in multiple $x y$ slices allowing for the volumetric reconstruction. For phase-averaged measurements, the SPIV (Stereoscopic Particle Image Velocimetry) system is triggered with the square signal used for the burst modulation. Image processing indicates peak values of high correlation, ensuring a good estimation of instantaneous velocities in the region of interest. No special filtering of the raw vector fields is undertaken. The 
parameters used for vector calculation of the SPIV data are set as follows: no image pre-processing, cross-correlation, multi-step with a size decrease of the interrogation window from $32 \times 32$ ( 2 passes) to $16 \times 16$ ( 2 passes), overlap of $50 \%$, and post-processing with median filter. The upper bound of uncertainty of instantaneous fields, calculated as in the work of Krebs et al., ${ }^{22}$ is close to $0.25 \mathrm{~m} \mathrm{~s}^{-1}$. In major-plane measurements $(x z), 500$ image pairs of instantaneous flow fields were acquired to compute a mean flow field. The time averaged flow fields acquired for the volumetric reconstruction are obtained with 340 image pairs per plane. In the phase-averaged measurements, 170 image pairs are used for each phase. Values are high enough to guarantee a satisfactory convergence.

\section{RESULTS}

\section{A. Unexcited jets}

In this section, we present results for the unexcited jet case. In this scenario, plasma actuators are not activated and the full flow field is reconstructed in 3D space, but not in time. The volumetric reconstruction corresponds therefore to the time averaged magnitudes, illustrating important properties of the jet mean flow. If the phenomenon to be observed in the near field were only $2 \mathrm{D}$, measurements of time-resolved PIV in a single slice would be enough to understand the flow physics. Being 3D, the analysis of the dynamics in the volume requires simultaneous (or phase averaged) information of what is occurring in an important number of slices. Before implementing any strategy to synchronize measurements at different planes, the first approach to describe the flow characteristics is to reconstruct the time-averaged 3D field. This filtered information is relevant because it bears the trace of the $3 \mathrm{D}$ jet dynamics and, in our scenario, reveals the existence of splitting, recomposition, and switching. Also, the time-averaged 3D field enables to contextualize the study through the comparison with previous works.

\section{Velocity flow fields in the major plane}

Contours of the velocity magnitude for different flow rates are presented in Fig. 4. We present both typical instantaneous velocity fields $U(x, y, z)$ and time averaged velocity fields $\langle U(x, y, z)\rangle$, in the symmetry plane containing the major axis of the slot $(y=0)$. For symmetry reasons, the time averaged velocity $\left\langle U_{y}\right\rangle$ has to be negligible on this plane. This provides a useful criterion that we used to determine the location of the plane $y=0$ precisely in the experiments.

As can be observed, the flow is quite symmetric with respect to the jet minor axis $(z=0)$, the centerline velocity decreases with the $x$ axis, and no significant expansion of the jet along the $z$ direction is observed (Fig. 4, right column). This behavior is typical of slender jets and is associated to the axis switching phenomenon. Traces of the instabilities developing close to the jet exit are visible in the instantaneous velocity fields (Fig. 4, left column). The filtering produced by the time averaging gives smoother fields, hiding this phenomenon.

A salient feature of both the instantaneous and time averaged velocity fields can be remarked: a region along $x$ is found where the velocity profiles present two off-axis peaks and a valley at the centerline (saddle back profile shape).

The analysis of the time averaged field at the symmetry plane $x z$ is particularly illustrative of the bifurcation phenomenon. Note that the velocity perpendicular to this symmetry plane is negligible and that, therefore, all inflows and outflows are contained in this plane. The peaks indicate the bifurcation of the inflow into two streams. As the velocity has a non-null value on the jet axis, the two streams are not completely separated. Further downstream, the positions of the off-axis peaks converge to a single maximum located again at the centerline $(z=0)$, indicating that the jet cores merge to recompose a single stream.

\section{Time averaged flow fields in the whole investigated region}

Results presented in this section are obtained processing the sets of the $36 x y$ slices of the whole investigated region. The case reported corresponds to a flow rate of $18 \mathrm{lpm}\left(300 \mathrm{~cm}^{3} \mathrm{~s}^{-1}\right)$, yielding 


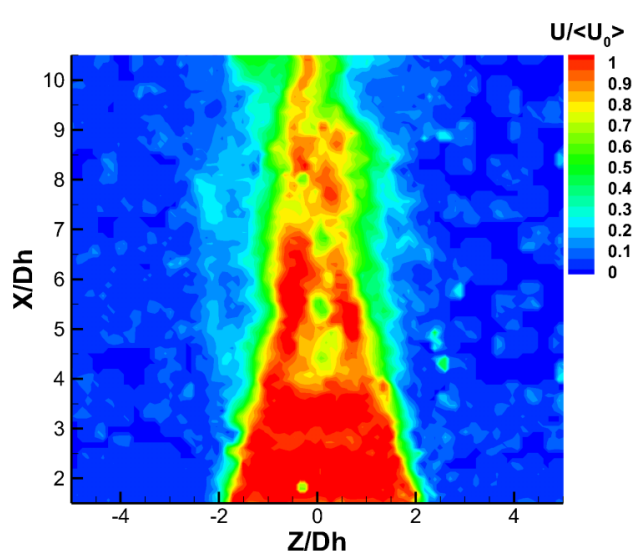

(a)

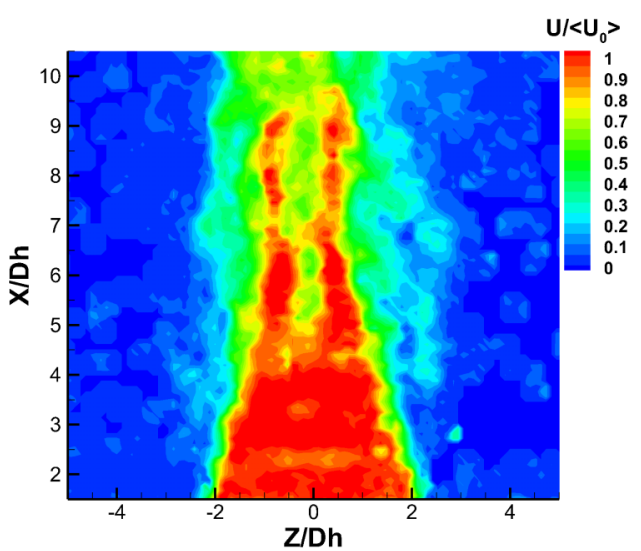

(c)

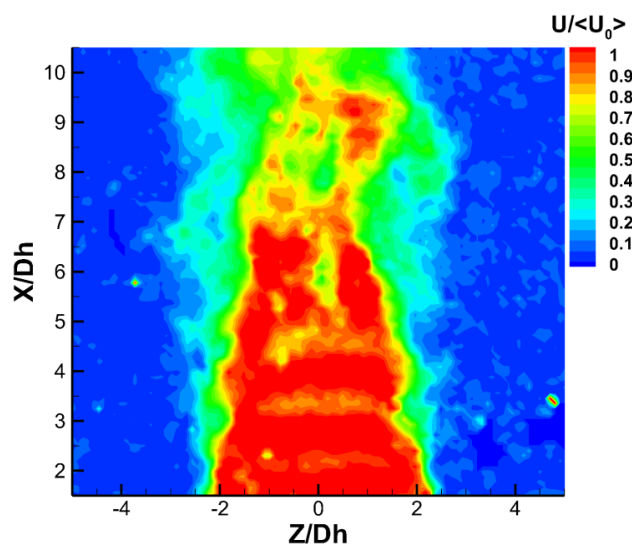

(e)

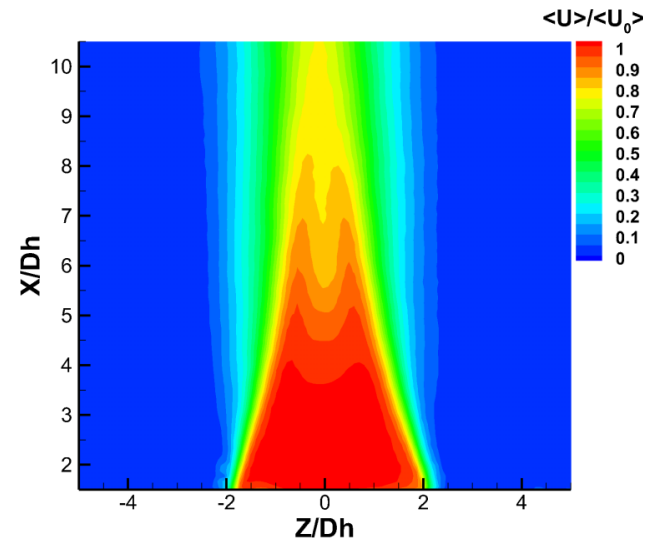

(b)

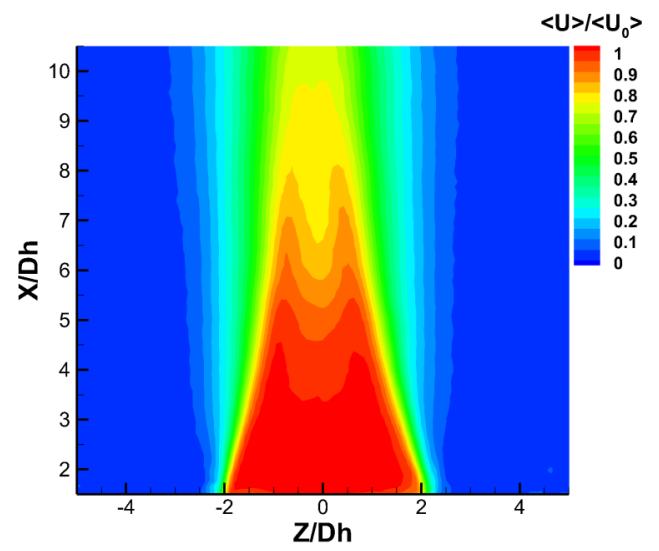

(d)

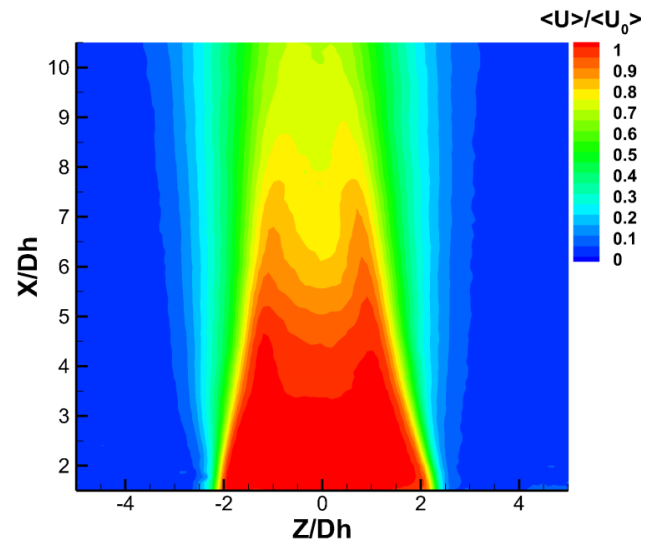

(f)

FIG. 4. Velocity magnitude fields at $y=0$ for different flow rates, normalized with mean exit velocities $\left\langle U_{0}\right\rangle$. Instantaneous fields are shown on the left (a), (c), and (e); and mean fields on the right (b), (d), and (f). Flow rate values are in (a) and (b) $22 \mathrm{lpm}\left(\left\langle U_{0}\right\rangle=12.2 \mathrm{~m} / \mathrm{s}, \operatorname{Re} \approx 2800\right)$, in (c) and (d) $24 \mathrm{lpm}\left(\left\langle U_{0}\right\rangle=13.3 \mathrm{~m} / \mathrm{s}, R e \approx 3100\right)$, and in (e) and (f) $26 \mathrm{lpm}$ $\left(\left\langle U_{0}\right\rangle=14.4 \mathrm{~m} / \mathrm{s}, R e \approx 3400\right)$.

an exit velocity of $\left\langle U_{0}\right\rangle=10 \mathrm{~m} \mathrm{~s}^{-1}$ and a Reynolds number (based on the hydraulic diameter $D_{h}$ ) of 2300. The jet behavior of this case is illustrative of the entire set of experiments performed in this study.

The iso-surface of the mean velocity magnitude of the jet in the bifurcation region is illustrated in Fig. 5. Three slices are shown, with the contour maps of the normalized mean streamwise velocity 


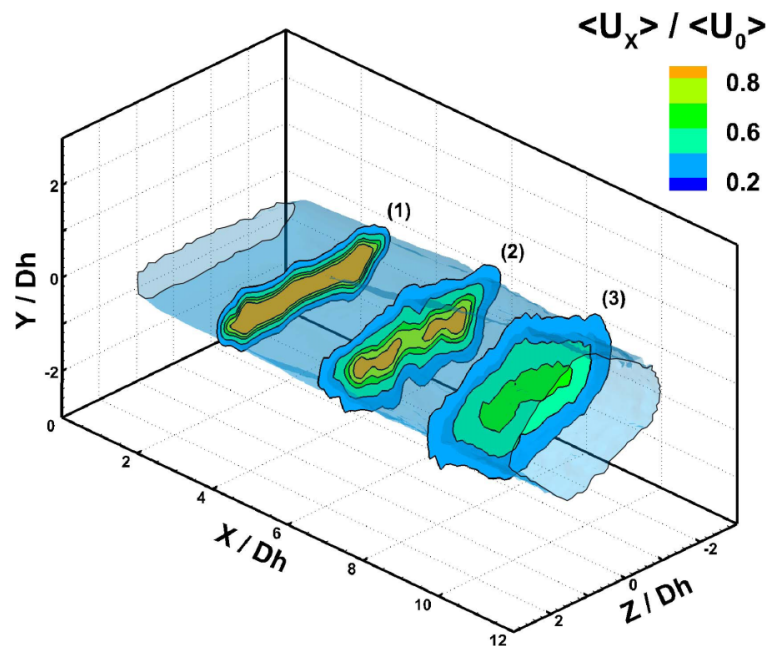

FIG. 5. Bifurcation region for the case with $\left\langle U_{0}\right\rangle=10 \mathrm{~m} \mathrm{~s}^{-1}(R e \approx 2300)$. Iso-surface of the mean velocity magnitude $\langle U\rangle=4 \mathrm{~m} \mathrm{~s}^{-1}$ in the bifurcation region combined with contour maps of the mean streamwise velocity $\left\langle U_{x}\right\rangle$, normalized with the mean exit velocity $\left\langle U_{0}\right\rangle$ for three different $y z$ planes.

in the $y z$ plane for $x / D_{h}=3, x / D_{h}=6$, and $x / D_{h}=9$. Plane (1) shows the standard profile for a slender jet, and plane (2) shows a cross section typical of a jet bifurcating along $z$. In plane (3), the jet has regained a standard profile, and it has expanded in $y$ but has not yet switched axes. Further downstream, the major and minor axes effectively change their role.

Time averaged fields of the streamwise velocity at different $y z$ planes are detailed in Fig. 6. These plots are similar to those in Ref. 10 for a rectangular jet, in Ref. 4 for a tapered slot jet, or in Ref. 23 for a synthetic jet issuing from a rectangular slot.

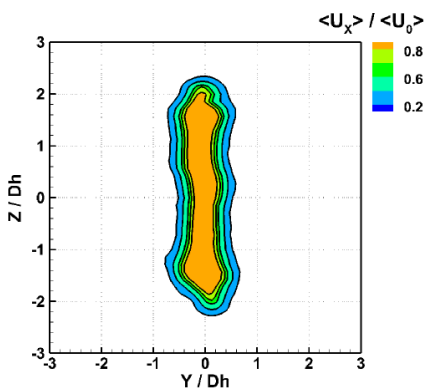

(a)

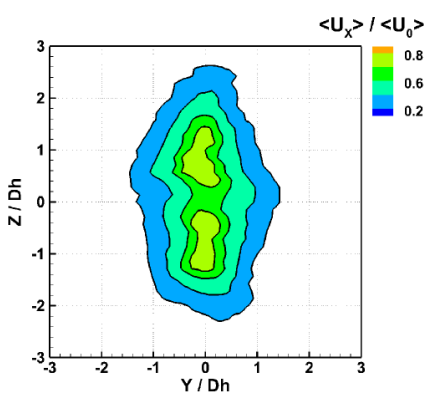

(d)

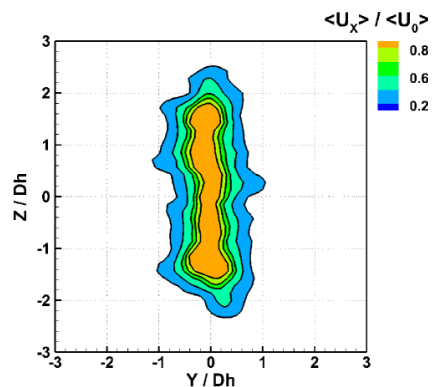

(b)

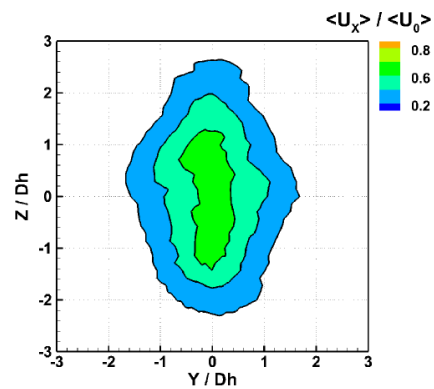

(e)

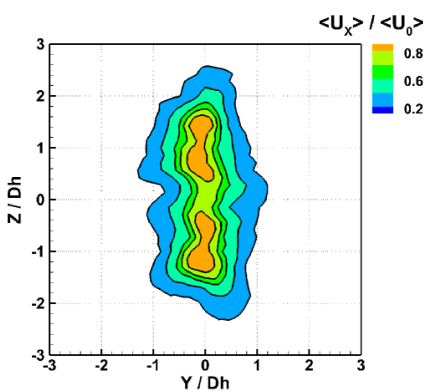

(c)

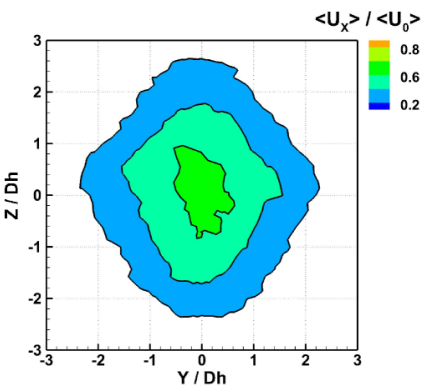

(f)

FIG. 6. Contour maps of the mean streamwise velocity $\left\langle U_{x}\right\rangle$, normalized with the mean exit velocity $\left\langle U_{0}\right\rangle=10 \mathrm{~m} \mathrm{~s}^{-1}$, for different $y z$ planes $\left(R e \approx 2300\right.$ ). (a) $x / D_{h}=3$. (b) $x / D_{h}=4.5$. (c) $x / D_{h}=6$. (d) $x / D_{h}=7.5$. (e) $x / D_{h}=9$. (f) $x / D_{h}=11$. 


\section{Axis-switching process}

The main interest of this subsection is to characterize the axis-switching of a jet that has undergone bifurcation upstream. Two questions naturally arise: how far apart are the locations of bifurcation and axis-switching? Does the bifurcation produce alterations in the jet properties?

Similarity analysis has been recently shown to be useful to quantify axis-switching in noncircular jets. ${ }^{24}$ The key in the analysis is to consider the similarity properties separately within the symmetry planes of the jet source. In the case of a slender jet, these symmetry planes are the major and minor planes. This kind of analysis was applied, for instance, to a pulsating jet, ${ }^{22}$ which was found to switch axes in accordance to in-plane similarity rules.

For non-circular nozzles, data are customarily normalized using the jet exit velocity $U_{0}$ and the hydraulic diameter $D_{h}$. Normalization with such scales yields non-collapsing velocity profiles. Instead, the in-plane similarity analysis uses one length scale per symmetry plane. The two length scales are the jet half-widths along $y$ and $z$, labeled $L_{y}$ and $L_{z}$. The values are shown in Fig. 7, together with the centerline velocity $U_{c}(x)$. In our experiment, the crossover position is located at $x / D_{h}=10.7$, after the bifurcation phenomenon.

By using the similarity variables $\left(y / L_{y}, z / L_{z}\right)$, the non-collapsing empirical profiles are found to rescale as collapsing profiles in the similarity variables within the $x$-range shown in Fig. 8 . The flow field is thus self-similar in the minor axis plane $(z=0)$ and the major axis plane $(y=0)$. The main finding that comes out of this discussion is that, even if the investigated jet has a strong vorticity dynamics close to the exit, the similarity properties constitute a robust flow feature that holds in spite of the occurrence of an upstream bifurcation.

\section{B. Excited jets}

To gain insight into the dynamics of the pre-switching bifurcation phenomenon, we resort to plasma actuators (Section II B). Using an excitation with a frequency close to the one of the predominant instabilities of the shear layer of the jet, a lock-on process can be attained ${ }^{25,20}$ even for a low perturbation amplitude. This allows for the phase-averaged reconstruction of the flow from data acquired at different $x y$ planes. The dynamics of the 3D vortical structures can be unveiled by suitably combining the results of the different $x y$ slices. The amplitude of the perturbation must however be set to allow for lock-on without substantially altering the natural base flow. With this constraint,

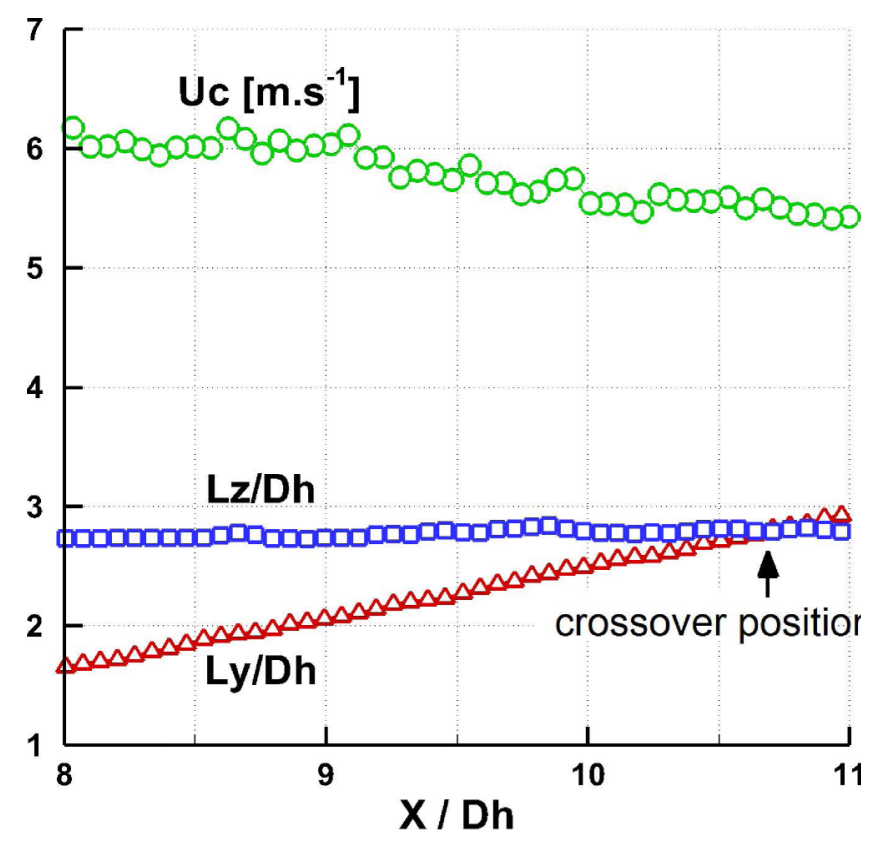

FIG. 7. Experimental values for the centerline velocity $U_{c}$ and the normalized jet half-widths $L_{y} / D_{h}$ and $L_{z} / D_{h}$. 


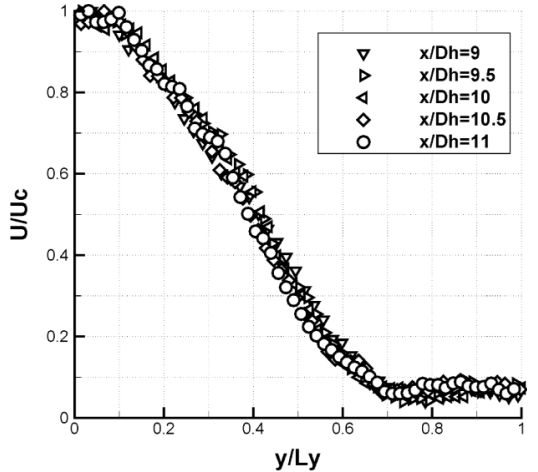

(a)

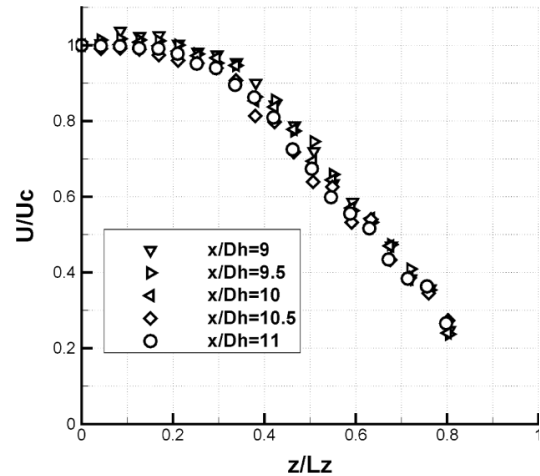

(b)

FIG. 8. Similarity analysis for the slender jet within $9<x / D_{h}<11$ at $R e \approx 2300$. Collapsing profiles with the rescaled variables in the minor plane (a) and in the major plane (b).

excitation should only organize the inherent coherent structures of the flow and remove the natural randomness of the jet.

\section{Phase-averaged flow fields in the whole investigated region}

Plasma actuators offer several advantages: the possibility of a localized action (a few millimeter around the edge of the air exposed electrode), together with a great versatility (high range of amplitudes and frequencies for the perturbations that are operated through the driven electrical signal).

To determine the adequate electrical parameters required to induce the lock-on phenomenon, a parametric study is undertaken by changing the amplitude $\mathrm{V}_{H V}$ and the frequency $\mathrm{f}_{B M}$ of the burst modulation. The influence of the burst modulation amplitude is investigated in a range extending from $\mathrm{V}_{H V}=2 \mathrm{kV}$ to $\mathrm{V}_{H V}=6 \mathrm{kV}$. The lower limit of this range corresponds to an amplitude that enables uniform plasma ignition. The excitation frequency is determined by changing the burst frequency $\mathrm{f}_{B M}$ and by identifying the excitation that requires a minimum amplitude to achieve lock-on. A summary of this parametric study is provided through four cases: (a) no actuation, (b) steady actuation at $\mathrm{V}_{H V}=2.5 \mathrm{kV}$, (c) actuation with a burst modulation at $\mathrm{V}_{H V}=2.5 \mathrm{kV}, \mathrm{f}_{B M}=1 \mathrm{kHz}$, and (d) with a burst modulation at $\mathrm{V}_{H V}=4 \mathrm{kV}, \mathrm{f}_{B M}=1 \mathrm{kHz}$. For the last three cases, the frequency of the carrier sinusoidal signal is set at $\mathrm{f}_{H V}=8 \mathrm{kHz}$.

The contours of the time-averaged velocity magnitude and of the time-averaged $z$-vorticity in the minor plane are plotted in Fig. 9 for cases (a) and (b). These figures show that velocity and vorticity flow fields are quite similar in both cases. When the actuator is operated with this amplitude, the amount of momentum added to the flow produces no distinguishable effects on the base flow pattern.

The contours of the phase-averaged velocity magnitude and the phase-averaged $z$-vorticity in the minor plane are plotted in Fig. 9 for cases (c) and (d). When we consider a typical phase-averaged field of any of these cases, the distribution of the velocity shows a dynamics of intense velocity packets moving downstream. The $z$-vorticity contours, in coincidence with these packets, present pairs of strong counter-rotating vortices. This behavior depends on the frequency and the amplitude of the excitation. A clear lock-on phenomenon is observed when $\mathrm{f}_{B M} \sim 1 \mathrm{kHz}$ : actuation serves here only to produce, in the near field, the development of monochromatic instabilities so that the phase of the cycle can be well-established. When experiments are undertaken at the same amplitude of excitation, but with values of $\mathrm{f}_{B M}$ not close to $1 \mathrm{kHz}$, the phase-averaged velocity and vorticity distributions are similar to the ones in case (a) or (b).

On the contrary, results from experiments undertaken for large amplitude values (illustrated in Fig. 9(d)) indicate alterations of the base flow: the jet width becomes larger in comparison with all the other cases and vorticity distributions are substantially modified whatever the phase. Taking into account these results, we choose an electrical excitation amplitude of $\mathrm{V}_{H V}=2.5 \mathrm{kV}$ and a frequency of $\mathrm{f}_{B M}=1 \mathrm{kHz}$. With these settings, the phase-locked results can be considered as representative of 

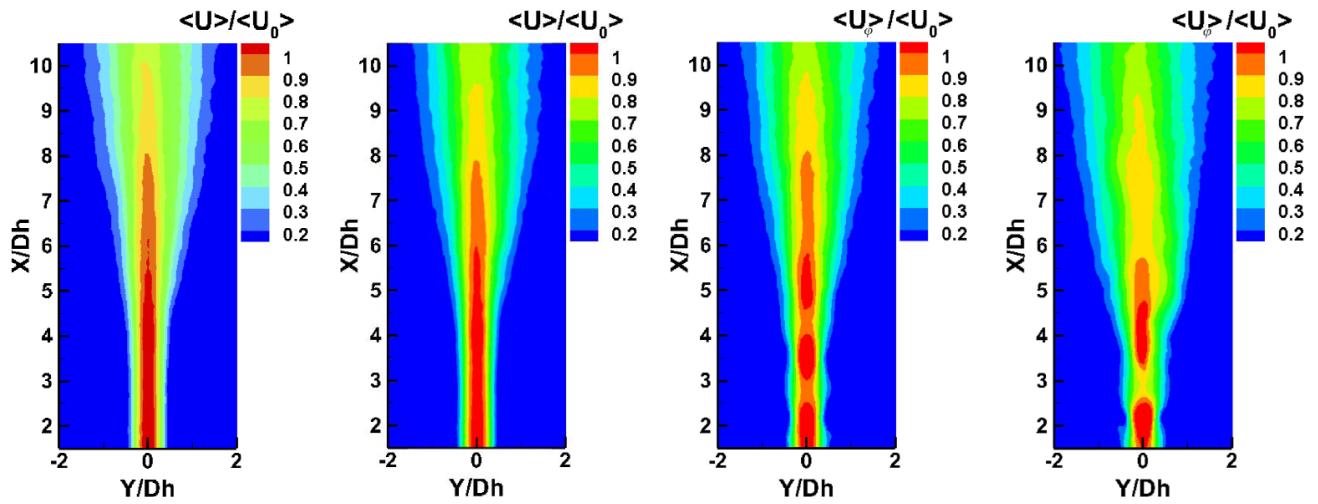

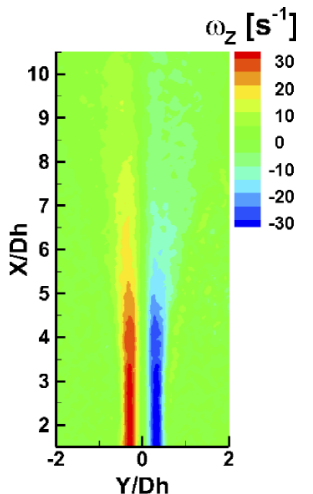

(a)

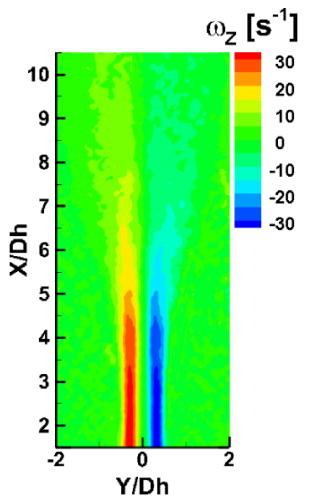

(b)

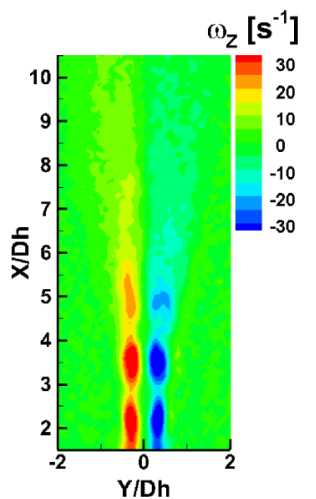

(c)

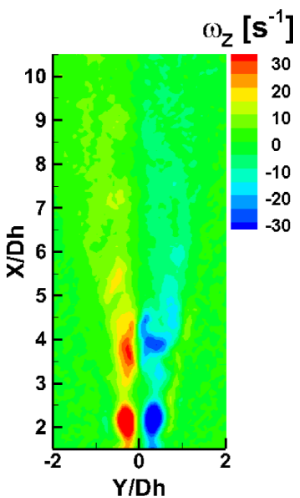

(d)

FIG. 9. Normalized velocity magnitude and $z$-vorticity in the minor plane for different actuation cases at $R e \approx 2300$ : timeaveraged values for cases (a) without actuation, (b) with continuous actuation at low amplitude $\left(\mathrm{V}_{H V}=2.5 \mathrm{kV}, \mathrm{f}_{H V}=8 \mathrm{kHz}\right)$. Phase-averaged values for cases, (c) burst modulation at low amplitude $\left(\mathrm{V}_{H V}=2.5 \mathrm{kV}, \mathrm{f}_{B M}=1 \mathrm{kHz}, \mathrm{f}_{H V}=8 \mathrm{kHz}\right)$, (d) burst modulation at high amplitude $\left(\mathrm{V}_{H V}=4 \mathrm{kV}, \mathrm{f}_{B M}=1 \mathrm{kHz}-\mathrm{f}_{H V}=8 \mathrm{kHz}\right)$.

the unexcited jet case. Notice that no Coanda effect is found in our study, since there are no traces of this phenomenon at plane $z=0$.

\section{Vorticity dynamics in the pre-switching region}

In this section, results of the plasma-synchronized fields are provided. To have access to the 3D characteristics of the flow, we process the phase averaged velocity fields over 36 series of $x y$ slices. This procedure provides a sufficiently dense volumetric grid to compute the phase-locked 3D vorticity fields without interpolations. Vortical structures, identified with the Q-criterion, are shown in Figs. 10 and 11.

Fig. 10 shows the structures of the jet for different phase angles. Fig. 11 shows three top views of the jet in the $y z$-plane for $\varphi=0^{\circ}$. These results reveal that, close to the jet exit, the vortical structures are aligned in two anti-parallel vortex tubes oriented along the $z$ axis. This is in agreement with the minor-plane phase-averaged $z$-vorticity measurements presented in Sec. III B 1 (Fig. 9(c)).

As these structures are advected downstream, the tubes deform both along the $z$ and the $y$ axes. In the $x z$ plane, vortex-tube waving is found for $x / D_{h} \geq 3$ (Fig. 10). The vortex tubes also deform in the $y z$ plane, approaching each other at their half-length in an "8-shape," for $x / D_{h} \sim 4.5$ (Fig. 11(c)). For $x / D_{h}>5$, coherent structures become more and more diffuse.

\section{Core splitting analysis}

Close to the nozzle, the jet is found to produce cyclically two opposite counter-rotating vorticity tubes oriented in the direction of the slot. These tubes are advected by the flow and tend to distort 


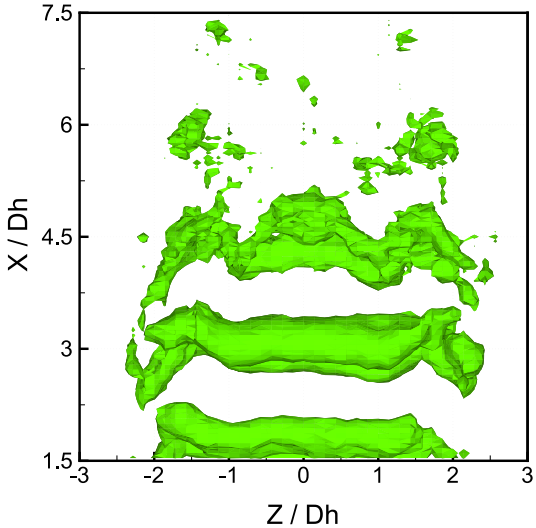

(a)

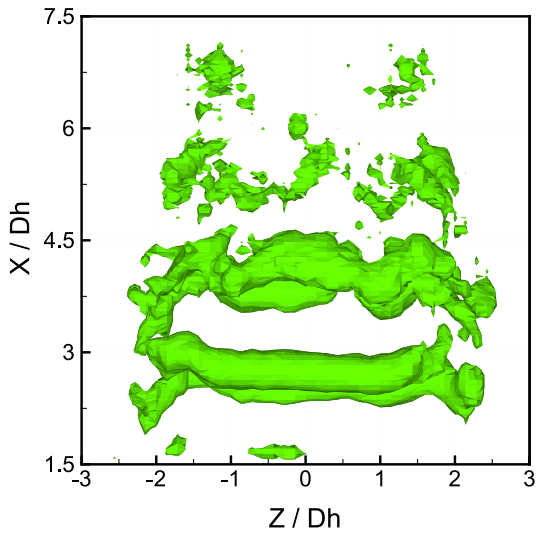

(c)

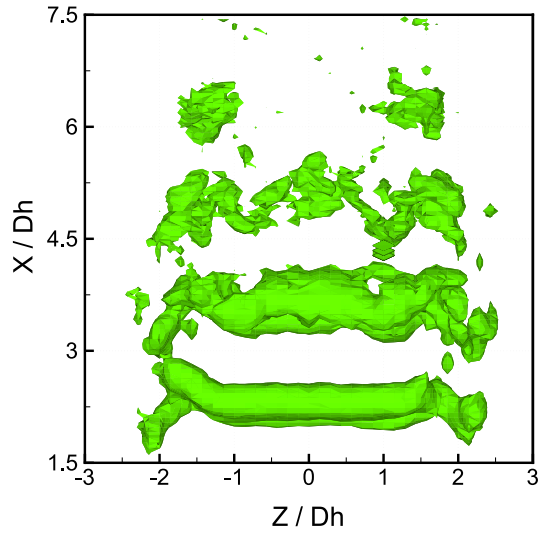

(b)

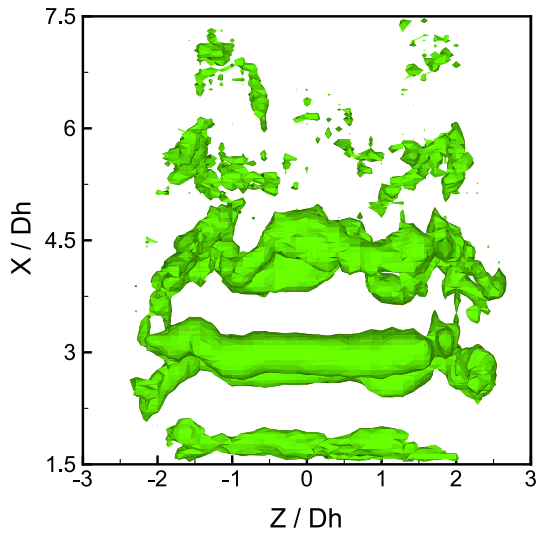

(d)

FIG. 10. Evolution of the vortical structures at $R e \approx 2300$, represented by the Q-criterion, for different phase values. (a) $\varphi=0^{\circ}$. (b) $\varphi=90^{\circ}$. (c) $\varphi=180^{\circ}$. (d) $\varphi=270^{\circ}$.

downstream. It is well known that two anti-parallel vortex tubes, when disposed sufficiently close to each other, tend to develop long wave instabilities if their vorticity strength is high enough. The development of this kind of instability, known as Crow instability, ${ }^{26}$ deforms the tubes, approaching them in some locations. This process may entail a reconnection of the tubes, accompanied by the creation of thin vortical structures. A linear instability analysis capable of describing the early stages of the phenomenon is available in the literature, for the cases in which the length of the tubes is much

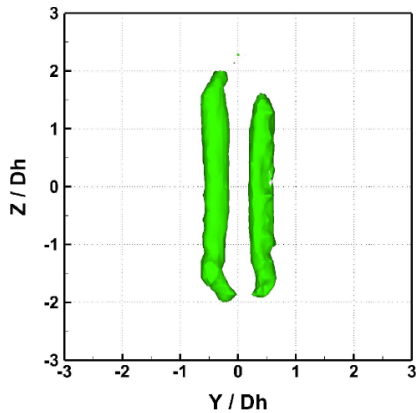

(a)

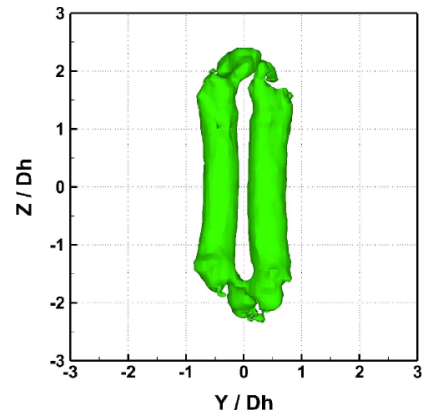

(b)

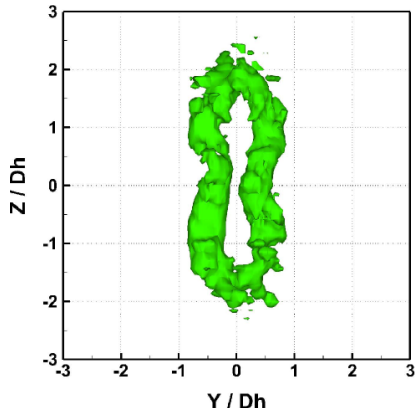

(c)

FIG. 11. Top view of the evolution of the vortical structures at $R e \approx 2300$, represented by the Q-criterion in the $y z$-plane, for a phase angle of $\varphi=0^{\circ}$. (a) $1.5 \leq x / D h \leq 2.25$, (b) $2.25 \leq x / D h \leq 3.75$ and (c) $3.7 \leq x / D h \leq 5.25$. 


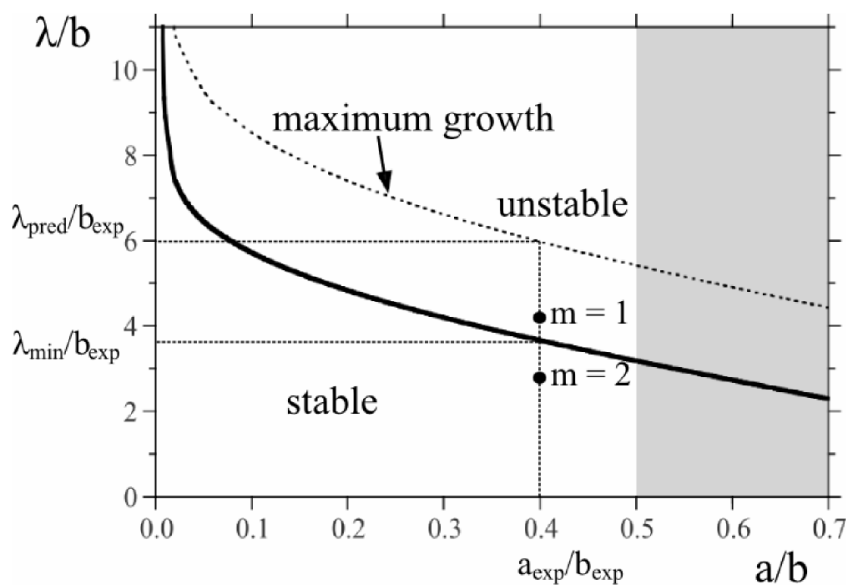

FIG. 12. Stability diagram for a pair of vortex tubes with $\lambda$ the instability wavelength, $a$ the radius of the tube cores, and $b$ the distance separating the tube centres. ${ }^{27}$ The region in gray has no physical significance since the cores are overlapping there.

larger than their radius. An arbitrary perturbation of the initially straight and parallel vortex tubes is decomposed into a set of normal modes. These modes consist of plane sinusoidal displacements characterized by their axial wavelength $\lambda$, by their orientation with respect to the line joining the initial vortex axes, and by their symmetry. The analysis leads to the identification of the most amplifying modes of the infinite-length tubes.

The stability diagram for the symmetric bending-wave perturbations of a vortex pair is provided in Fig. 12, extracted from Leweke and Williamson. ${ }^{27}$ In this graph, $a / b$ is the ratio between the radius of the tube cores $(a)$ and the distance separating the axes of the tubes $(b)$. In the limiting case $(a / b=0.5)$, the graph indicates $\lambda_{\text {min }} \simeq 3.15 b$ and $\lambda_{\text {pred }} \simeq 5.4 \mathrm{~b}$.

By fitting the vorticity profile of each tube with a Gaussian function, ${ }^{27}$ we can compute the parameters $a$ and $b$ corresponding to our experimental case in the instability diagram: the mean separation of the vortex tubes (plane (1) of Fig. 11) is $b_{\text {exp }} \simeq 2.23 \mathrm{~mm}$ and the mean core radius (equivalent Rankine vortex radius) is $a_{\text {exp }} \simeq 0.97 \mathrm{~mm}$, so that the ratio $a_{\text {exp }} / b_{\text {exp }} \simeq 0.4$. For all the tested flow rates, $a_{\text {exp }} / b_{\text {exp }}$ remains approximately coincident. For $a_{\text {exp }} / b_{\text {exp }} \simeq 0.4$, the instability sets in for wavelengths larger than $\lambda_{\min } \simeq 3.6 b_{\text {exp }}$. The predominant instability (that with the maximum growth rate) has, in this case, the value $\lambda_{\text {pred }} \simeq 6 b_{\text {exp }}$.

The vortex tubes of the jet that we study have a finite length $l$ that scales with the major side of the slot. This is not without consequence on the wavelength of the instability that can develop in our scenario, where $\lambda$ should comply with the following symmetry condition:

$$
\frac{l}{\lambda}=m+\frac{1}{2} \quad \text { with } \quad m \in \mathbb{N}
$$

stating that the instability should have an integer plus a half wavelength along the slot. The instability mode with an "8-shape" corresponds to $m=1$, which represents the dual core splitting responsible for the saddle-back profile observed in Section III A 2. Mode $m=0$ would correspond to a single core jet (no saddle-back profile) and $m>1$ would correspond to multiple core splitting.

We associate the distortion of the vortex tubes (and therefore the bifurcation observed in our experiments) to the development of the Crow instability for finite length tubes. In such case, the wavelengths associated to the process should be close to the ones with maximum growth rate predicted by this theory. A first approach to the understanding of the instabilities that develop in the case of anti-parallel vortex tubes with a finite length can be obtained using as reference the instability diagram of Fig. 12, combined with Equation (1). With this rationale, near-field bifurcation for a slender jet should be expected to occur for $m$ values satisfying $\lambda / b \sim \lambda_{\text {pred }} / b$. If we consider that $l$ approximately matches the length of the major side of the slot, Fig. 12 shows that the condition is met for $m=1$. This result is in agreement with the "8-shape" observed in Fig. 11(c). 
In addition, this reasoning can be used to estimate the minimum aspect-ratio for which bifurcation should be expected. Even if this threshold is known to be geometry-dependent, there is experimental evidence that this value is generally close to five. ${ }^{10}$ Let us consider the limiting case of the instability diagram $(a / b=0.5)$, for which $\lambda_{\min }=3.15 b$. With $b$ approximated by the minor side of the slot and $l$ by the major side of the slot, the aspect ratio is $l / b$. Considering Equation (1), we can conclude that the instability promoting jet bifurcation $(m=1)$ should develop for aspect ratios greater than $3.15 \cdot 3 / 2=4.725$, a value lying close to 5 .

Note also that keeping $a / b$ fixed at the value of the case that we study, a moderate increase of the aspect ratio would produce an increase of the growth rate of the perturbation corresponding to $m=1$. If the aspect ratio is further increased, the mode $m=2$ may become predominant and produce multiple core splitting. In both cases, this will redound in an increase of the near field mixing as observed in rectangular jets when slot aspect ratio is increased. ${ }^{28}$

In summary, in spite of its simplicity, this approach appears as capable of providing a reasonable explanation for long-standing observations concerning slender jets.

\section{CONCLUSION}

This work studies the 3D flow physics of a slender jet exiting from a simple constriction with the form of two semi-cylindrical lips. The analysis is performed with data issued from stereoscopic PIV measurements considering time-averaged magnitudes and phase-averaged fields synchronized with plasma actuators.

The volumetric reconstruction of the full mean flow field of the investigated jet discloses the characteristics of the complex phenomena occurring in the region close to the slot exit: the splitting of the jet into two streams that merge before axis-switching. The velocity field analysis illustrates this phenomenon through the presence of two maxima of the jet velocity that are symmetrically disposed at both sides of the centerline along the major axis direction. Downstream, the jet regains a single core profile, with only one maximum located at the jet centerline. Axis-switching occurs downstream in accordance with self-similarity rules holding for slender jets. This property is quite robust since it persists in spite of the complex phenomena observed upstream.

An explanation of the jet core splitting is provided in terms of the symmetric Crow instability. A rough extension of this theory is proposed to incorporate the finite size of the vortex tubes retrieved close to the slot exit. For this mechanism to be relevant in the near field dynamics, the nozzle must produce vortex tubes with an appropriate size and separation. These requirements are fulfilled in the configuration we study. Being linear, this theory can only predict the first stages of the instability development, throwing no light on the occurrence of ulterior vortex tube reconnection processes or on subsequent vortex ring formation. Yet, the analysis satisfactorily predicts the main characteristics of the vortex tube distortion associated to the jet core splitting and provides an estimation of the minimum aspect ratio required for bifurcation to occur, producing a threshold value that coincides with observations in previous reports. Of course, exhaustive parametric studies are necessary to better ponder the mechanisms driving the bifurcation in different scenarios. In the case of jets issued from nozzles with complex configurations, the link between the nozzle geometry and the vortex tube configuration (length, radius, and separation) should be particularly understood. An oncoming study based on high performance numerical simulations is likely to provide further insight into the time scales involved in the different processes as well as on the vortex dynamics of the jet recomposition.

${ }^{1}$ H. U. Riisgard, B. H. Jorgensen, K. Lundgreen, F. Storti, J. H. Walther, K. E. Meyer, and P. S. Larsen, "The exhalant jet of mussels mytilus edulis," Mar. Ecol.: Prog. Ser. 437, 147-164 (2011).

${ }^{2}$ B. D. Erath and M. W. Plesniak, "Impact of wall rotation on supraglottal jet stability in voiced speech," J. Acoust. Soc. Am. 129(3), EL64-EL70 (2011).

${ }^{3}$ C.-M. Ho and E. Gutmark, "Vortex induction and mass entrainment in a small-aspect-ratio elliptic jet," J. Fluid Mech. 179 383-405 (1987).

${ }^{4}$ E. J. Gutmark and K. C. Schadow, "Flow characteristics of orifice and tapered jets," Phys. Fluids 30, 3448-3454 (1987).

${ }^{5}$ W. R. Quinn, "On mixing in an elliptic turbulent free jet," Phys. Fluids 1(10), 1716-1722 (1989).

${ }^{6}$ E. J. Gutmark and F. F. Grinstein, "Flow control with noncircular jets," Annu. Rev. Fluid Mech. 31, 239-272 (1999).

${ }^{7}$ W. C. Reynolds, D. E. Parekh, P. J. D. Juvet, and M. J. D. Lee, "Bifurcating and blooming jets," Annu. Rev. Fluid Mech. 35, 295-315 (2003). 
${ }^{8}$ F. Hussain and H. S. Husain, “Elliptic jets. Part I. Characteristics of unexcited and excited jets," J. Fluid Mech. 208, 257-320 (1989).

${ }^{9}$ F. F. Grinstein, "Self-induced vortex ring dynamics in subsonic rectangular jets," Phys. Fluids 7, 2519-2521 (1995).

${ }^{10}$ A. P. Vouros, T. Panidis, A. Pollard, and R. R. Schwab, "Near field vorticity distributions from a sharp-edged rectangular jet,” Int. J. Heat Fluid Flow 51, 383-394 (2015).

${ }^{11}$ L. J. S. Bradbury, “The structure of a self-preserving turbulent plane jet," J. Fluid Mech. 23, 31-64 (1965).

12 Y. Tsuchiya, C. Horikoshi, and T. Sato, "On the spread of rectangular jets," Exp. Fluids 4, 197-204 (1986).

${ }^{13}$ X. Pelorson, A. Hirschberg, R. V. Hasselt, A. Wijnands, and Y. Auregan, "Theoretical and experimental study of quasisteadyflow separation within the glottis during phonation. Application to a modified two-mass model," J. Acoust. Soc. Am. 96(6), 3416-3431 (1994).

${ }^{14}$ X. Pelorson, A. Hirschberg, A. P. J. Wijnands, and H. Baillet, "Description of the flow through in-vitro models of the glottis during phonation,” J. Acoust. Soc. Am. 96, 3416-3431 (1995).

${ }^{15}$ L. Bailly, X. Pelorson, N. Henrich, and N. Ruty, "Influence of a constriction in the near field of the vocal folds: Physical modeling and experimental validation," J. Acoust. Soc. Am. 124, 3296-3308 (2008).

${ }^{16}$ N. E. Chisari, G. Artana, and D. Sciamarella, "Vortex dipolar structures in a rigid model of the larynx at flow onset," Exp. Fluids 50, 397-406 (2011).

17 J. R. Roth, D. M. Sherman, and P. S. Wilkinson, "Electrohydrodynamic flow control with a glow-discharge surface plasma," AIAA J. 38, 1166-1172 (2000).

${ }^{18}$ E. Moreau, "Airflow control by non-thermal plasma actuators," J. Phys. D: Appl. Phys. 40, 605 (2007).

${ }^{19}$ T. C. Corke, C. L. Enloe, and S. P. Wilkinson, "Dielectric barrier discharge plasma actuators for flow control," Annu. Rev. Fluid Mech. 42, 505-529 (2010).

${ }^{20}$ T. N. Jukes and K. S. Choi, "Flow control around a circular cylinder using pulsed dielectric barrier discharge surface plasma," Phys. Fluids 21, 084103 (2009).

${ }^{21}$ P. Audier, H. Rabat, A. Leroy, and D. Hong, "Experimental investigation of a surface DBD plasma actuator at atmospheric pressure in different $\mathrm{N}_{2} / \mathrm{O}_{2}$ gas mixtures," Plasma Sources Sci. Technol. 23, 065045 (2014).

${ }^{22}$ F. Krebs, F. Silva, D. Sciamarella, and G. Artana, "A three-dimensional study of the glottal jet," Exp. Fluids 52, 1133-1147 (2012).

${ }^{23}$ B. L. Smith and A. Glezer, "The formation and evolution of synthetic jets," Phys. Fluids 10(9), 2281-2297 (1998).

${ }^{24}$ D. Sciamarella, F. Silva, and G. Artana, "Similarity analysis of a glottal-like jet," Exp. Fluids 53(3), 765-776 (2012).

${ }^{25}$ J. Wu, X. Lu, A. Denny, M. Fan, and J. Wu, "Post-stall flow control on an airfoil by local unsteady forcing," J. Fluid Mech. 371, 21-58 (1998).

${ }^{26}$ S. C. Crow, "Stability theory for a pair of trailing vortices," AIAA J. 8, 2172-2179 (1970).

${ }^{27}$ T. Leweke and C. H. K. Williamson, "Experiments on long-wavelength instability and reconnection of a vortex pair," Phys. Fluids 23, 024101 (2011).

${ }^{28}$ W. R. Quinn, “Turbulent free jet flows issuing from sharp-edged rectangular slots: The influence of slot aspect ratio," Exp. Therm. Fluid Sci. 5, 203-215 (1992). 